\title{
L'industrialisation des médias chinois dans le contexte de la mondialisation
}

Jinhai Zhang

\section{(2) OpenEdition}

1 Journals

Édition électronique

URL : http://journals.openedition.org/communicationorganisation/2935

DOI : 10.4000/communicationorganisation.2935

ISSN : $1775-3546$

Éditeur

Presses universitaires de Bordeaux

Édition imprimée

Date de publication : 1 mai 2004

ISSN : 1168-5549

Référence électronique

Jinhai Zhang, "L'industrialisation des médias chinois dans le contexte de la mondialisation », Communication et organisation [En ligne], 24 | 2004, mis en ligne le 27 mars 2012, consulté le 01 mai 2019. URL : http://journals.openedition.org/communicationorganisation/2935; DOI : 10.4000/ communicationorganisation.2935

Ce document a été généré automatiquement le 1 mai 2019.

(c) Presses universitaires de Bordeaux 


\title{
L'industrialisation des médias chinois dans le contexte de la mondialisation
}

\author{
Jinhai Zhang
}

\section{Contexte et motivation}

1 L'industrialisation des médias chinois a débuté par deux grandes affaires, lin 1978 le Quotidien du Peuple et les sept journaux pékinois ont adressé conjointement au Ministère des Finances une demande pour bénéficier de la gestion de l'économie du marché accordée aux établissements d'utilité publique. L'autre, c'est que le marché publicitaire a été ouvert au mois de janvier 1979. Celle-ci pousse beaucoup à la réalisation de l'industrialisation des médias tandis que celle-là essaie de chercher un support politique et une affirmation officielle pour l'orientation vers le marché des médias chinois.

2 Auparavant, les médias chinois étaient toujours un moyen d'expression idéologique et un intermédiaire transmettant au public des messages officiels: indemnisés par les ressources publiques, ils relevaient du secteur de l'idéologie : en un mot. Ils étaient des sociétés administratives et cela n'avait pas changé depuis la fondation de la République Populaire de Chine. Mais il $\mathrm{y}$ a eu des changements dans la vie politique de la Chine à la fin des années $70:$ la révolution de la libération de l'esprit à l'échelle du territoire national après la Grande Révolution Culturelle, la proposition de la Réforme et de l'Ouverture ainsi que lu stratégie du développement national autour de la construction de l'économie entrainent l'évolution du domaine des médius. Les scientifiques ont commencé à réfléchir sur la fonction multiple des médias et de leur contribution à la construction économique. En fait, l'industrialisation des médias chinois est un nouvel acte de la transmission et de la communication accompagnant la construction de l'économie et la mise en marche de la Réforme et de l'Ouverture. Non seulement cette démarche a été couronnée de succès mais elle a aussi lié les médias chinois à ceux du monde entier. 
3 La Réforme et l'Ouverture de la Chine et le transfert à la construction de l'économie offrent une opportunité aux médias chinois pour leur industrialisation, correspond aux propres besoins des médias chinois.

4 Avec l'ancien système d'indemnisation, les médias chinois se forçaient à tenir un équilibre entre les dépenses et les remboursements, ils ne s'en sortaient jamais et ne voyaient pas le bout du tunnel. Après la $3^{\text {eme }}$ réunion du $11^{\mathrm{eme}}$ Congés du parti Communiste Chinois, guidés par l'objectif de construction de l'économie, les médias chinois ont essayé de concevoir dans leur esprit un nouveau système de financement répondant à notre temps.

\section{Evolution de l'industrialisation}

En 1978. le Ministère des Finances a ratifié l'accord permettant au Quotidien du Peuple de bénéficier de la gestion de l'économie du marché accordée aux établissements d'utilité publique En avril de l'année suivante, le ministère a permis la mise à l'épreuve des clauses de fonds de la société des groupes de presse pour assurer la gestion de société de l'économie du marché en matière financière.

$6 \quad$ Le 4 janvier 1979. Le Quotidien du Peuple a pris l'initiative de remettre en état la publicité commerciale. Le Quotidien de la libération et Le Journal Wenhui lui ont emboîté le pas. Par la suite, le Ministère de la communication et de l'information ont approuvé formellement cette démarche. C'était les premiers pas de l'industrialisation de la presse.

7 Si l'on jette un regard en arrière sur l'évolution de l'industrialisation des médias chinois, on ne peut pas oublier les faits ci-dessous :

8 En juillet 1984 l'Association des Journalistes Chinois a organisé la première réunion sur la gestion de l'exploitation de la presse après la Révolution Culturelle.

$9 \quad$ En 1985. Le Quotidien de Luoyang a été tonde en Henan avec ses propres capitaux : en 1999 les journaux de cette sorte, fonctionnant sans aide de l'État, constituaient ensemble un tiers des titres de presse.

En 1985 la Chine possédait 188 groupes de presse.

11 En 1987 le Comité des sciences de l'Etat a publié pour la première fois le bilan de la classification des industries, et a classé l'industrie de l'Information et l'industrie de la Radio et de la Télévision dans le secteur de la production informatique.

En 1987. Le Quotidien de Cantai a été le premier à augmenter sa pagination.

En 1988. le Bureau d'administration de l'information et le Bureau de la gestion de l'industrie et du commerce ont conjointement promulgué les règlements sur les services à titre onéreux et ses activités de l'exploitation des journaux, des périodiques et des maisons d'édition.

En septembre 1992. la Conférence d'échange des expériences de la gestion de la presse dirigée par la Confédération de la Presse Chinoise s'est tenue dans la province de Jiangxi. La conception de l'économie de l'industrie de presse a vu le jour.

fin 1993. le Conseil des Affaires d'État a promulgué le décret de définissant le secteur tertiaire et a classé la gestion de la presse dans ce secteur.

En 1993. le Bureau de la recherche de l'information de l'Académie Chinoise s'est chargé de l'organisation du séminaire sur l'économie du marché socialiste et de l'industrie de 
l'information. Désormais, la polémique sur les médias chinois est terminée et l'industrialisation des médias chinois s'est développée pas à pas et en douceur. Elle s'avançait lentement et avait des difficultés à sortir des contraintes de l'ancien système. Par conséquent, elle avait besoin d'un soutien officiel. Mais, en raison du contrôle strict du gouvernement, une progression douce et prudente fut décidée.

À travers tous les phénomènes, on pourrait remarquer une approbation directe et indirecte venant $d u$ gouvernement: l'autorisation $d u$ ministère sur les activités d'exploitation de la publicité de la presse et la permission des services à titre onéreux étaient bien délivrées par des décrets ministériels. Quant au macro politique, le classement de l'industrie de l'information, de la radio et de la télévision dans le secteur de la production informatique, témoignent d'une attitude positive du gouvernement..

On dit que la pratique doit toujours être guidée par la théorie. Mais la théorie des médias chinois est bien loin de la pratique. De la Révolution Culturelle jusqu'en 1992 les milieux scientifiques se sont focalisés sur la critique des idées traditionnelles de l'information et la reconnaissance de la fonction multiple des médias. Dès 1993 les scientifiques ont traité le sujet des caractéristiques des médias. Cela donne des points d'appui au développement ultérieur des médias. Cette discussion a continué jusqu'à la fin des années 90.

Si l'on dit que de 1978 à 1993 c'était une période d'essai, à partir de 1993. avec l'approbation de la politique cl le support de la théorie, l'industrialisation est entrée dans une période de pratique et de victoire.

Il faut encore dire que c'est la presse qui a pris l'initiative du processus de celte révolution. En fait, la radio et la télévision l'ont suivie de très près. À cause du contenu de divertissement qui constitue une de leurs particularités, leurs actions n'ont pas provoqué la même attention du public que la presse.

\section{Signification et problèmes à résoudre}

21 Avant les révolutions dans le secteur des médias, les médias chinois étaient toujours indemnisés par les ressources publiques. Ils ne sortaient pas de ce système rétrograde par manque de capitaux ils ne pouvaient pas progresser. Après le commencement de l'industrialisation l'État a progressivement diminué, voire annulé, les indemnités et les aides financières. Les Médias ont bénéficié des revenus de leur propre exploitation. Une Fois les charges acquittées, ils ont pu investir pour le développement.

En réalité, le fonctionnement de l'industrialisation est dû au problème budgétaire des années 70. À la suite de la Grande Révolution Culturelle, l'économie nationale était proche de l'effondrement. Cent choses laissées a l'abandon étaient à remettre en état. L'Etat ne pouvait pas paver les dépenses des médias, C'est pourquoi la proposition du Quotidien $d u$ Peuple fut si facilement acceptée par le gouvernement. Cela témoigne d'un compromis du politique avec le financier. On observe des cas semblables au début de la fondation de la nouvelle Chine.

En 1978 il \avait 186 journaux. 930 magazines. 93 stations de radio cl 32 de télévision. En 2001 on possédait 2111 journaux. 8889 magazines. 301 stations de radio. 357 de télévision. Prenons l'exemple de la presse : en 1978. les 186 journaux totalisaient un tirage de 42.8 million d'exemplaires, soit en total 11.35 milliards de pages: en 2001 les 2111 journaux avaient un tirage de 181.3 million d'exemplaires, soit une totalité de 
93.89 milliard de pages. ${ }^{1}$ Si l'on n'avait pas mis en marche l'industrialisation, une telle évolution eût été impossible.

D'après les statistiques, actuellement, plus de $90 \%$ des médias ont déjà réussi à équilibrer les charges et les revenus : moins de $10 \%$ de la presse relevant du PCC bénéficient encore des indemnités administratives. La plupart des entreprises de médias s'appuient sur leurs propres forces et sont devenues de grands contributeurs en termes de fiscalité.

Ayant connu de bons résultats, ce secteur connaît quand même des problèmes dans le domaine de la structure industrielle, de l'assignation des objectifs et du fonctionnement. En plus, les petits médias se dispersent, ils sont incapables de lutter contre les risques du marché, n'arrivent pas à l'économie d'échelle. L'échec est au niveau de l'exploitation multiple dans des secteurs trop nombreux, la gestion trop routinière mène à une impasse: à cause d'une difficulté d'harmonisation des deux systèmes, l'ancien et le nouveau, le fonctionnement et la gestion restent routinières.

La plus importante question reste : comment réaliser l'industrialisation?

\section{Réponse : la concentration horizontale} chemin inévitable. Mais le problème est que la dispersion des petits médias et la faiblesse des médias individuels nécessitent l'intervention du gouvernement. Certains spécialistes estiment que la révolution de l'exploitation est réalisée passivement par la pression de l'économie, mais les actions suivantes manifestent la reconnaissance et l'adaptation du marché. On pourrait croire que l'industrialisation chinoise dans le secteur de médias confirme cette opinion.

35 Ce sont des phénomènes existant dans la période primaire de l'industrialisation de médias chinois. Quand la Chine aura l'expérience nécessaire sur les médias à diffusion à l'échelle du pays, la concentration horizontale sera obligatoire. Réellement, la naissance 
des sociétés de presse trans-régionales est un symbole important. En revanche, avec la circonstance spécifique de la Chine, le $\backslash$ rai essor de l'industrialisation impose des efforts.

La réalisation de l'industrialisation et de la concentration dans le secteur de médias dans le contexte de mondialisation, aidera les médias chinois à être en concurrence avec les médias mondiaux.

\section{BIBLIOGRAPHIE}

Association pour la Gestion et l'Exploitation des Journaux de la Chine. Gestion de l'exploitation de la presse. 1999

Bureau de l'Administration Nationale des Statistiques de la Chine. Annuaire des statistiques de la Chine. Maison d'édition de statistiques de la Chine. 2002

DONG Tiance. Fonctionnement industriel de la presse chinoise. Maison d'édition populaire de Sichuang. 2002

FANG. Hanqi. Histoire de l'information de la Chine. Maison d'édition de l'Université populaire de Pékin. 1999

HUANG. Shenmin. DING. Junjie. Recherche sur l'industrialisation et l'exploitation des médias chinois. Maison d'édition de l'Institut de la radiodiffusion de Pékin. 1997

YU. Guoming. Les prévisions au sujet du développement de l'industrialisation des médias chinois pendant les cinq prochaines années. Réseaux scientifiques des médias, forum des spécialistes. 2001 /HANG. Jinhai, L'industrialisation des médias et l'exploitation de la publicité. Marketing, diffusion, publicité. Maison d'édition de l'institut de la radiodiffusion de Pékin. 2001

\section{NOTES}

1. Ces chiffres ne prennent pas en compte les médias de Hongkong, de Taiwan et de Macao. 